\title{
NOETHER'S THEOREM FOR HOPF ORDERS IN GROUP ALGEBRAS
}

\author{
DAVID M. WEINRAUB
}

\begin{abstract}
Let $K$ be a local field with valuation ring $R$ of residue characteristic $p$ containing a primitive $p$ th root of unity $\zeta_{p}$. We state an analog to Noether's Theorem for modules over $R$-Hopf algebras and use induction techniques to deduce a criterion for this analog to hold. We then construct a family of noncommutative Hopf algebras which satisfy the criterion.
\end{abstract}

\section{INTRODUCTION}

If $L / K$ is a Galois extension of number fields with group $G$ and rings of integers $S / R$, then in a natural way, $L$ is a $K G$-module and $S$ is an $R G$ module. The Normal Basis Theorem asserts that $L \cong K G$ as $K G$-modules. The analogous result does not generally hold for $S$ and $R G$, even after localizing $R$. In 1931, E. Noether [N] showed that locally (for all primes $p$ of $R$ ) $S \cong R G$ as left $R G$-modules if and only if the extension $L / K$ is tame (that is, for every prime $p$ of $R$, the ramification index of any prime $P$ of $S$ lying over $p$ is relatively prime to the characteristic of the residue field $R / p)$. In fact, the following are equivalent:

(1) $S \cong R G$, locally, as $R G$-modules.

(2) $L / K$ is tame.

(3) The trace map $S \rightarrow R$ is surjective.

(4) $S$ is a projective $R G$-module.

In 1986, L. Childs and S. Hurley $[\mathrm{C} / \mathrm{H}]$ generalized the notions of tameness and local normal basis to $H$-modules, where $H$ is an $R$-Hopf algebra. Their definitions specialized to those above when $H=R G$ and they obtained, in particular, the result that when $H$ is commutative, $S \cong H$ locally as $H$-modules if and only if $S$ is $H$-tame.

Waterhouse [W] notes that the Childs/Hurley notions of tameness do not imply the existence of local normal basis when $H$ is noncommutative, even for group rings over fields. The counterexample is not entirely satisfactory in that any "natural" number theoretic example would have normal basis over the quotient field, in accordance with a result of Kreimer and Cook [K/C].

Note that the Childs/Hurley results assume commutativity of $H$, yet Noether's Theorem holds for arbitrary finite groups. Accordingly, there seems reason to believe that analogous results should hold for (at least some) noncommutative $H$ (other than group algebras). We say that Noether's Theorem

Received by the editors June 30, 1991 and, in revised form, February 7, 1992.

1991 Mathematics Subject Classification. Primary 16A24, 19A22; Secondary 11S23.

Key words and phrases. Hopf algebras, induction. 
holds for a Hopf algebra $H$ (finitely generated and projective) over a discrete valuation ring $R$ with quotient field $K$ if the following are equivalent:

(1) $S$ is a projective $H$-module and $K \otimes S \cong K \otimes H$ as $K \otimes H$-modules.

(2) $S \cong H$ as $H$-modules.

The only implication of interest here is $(1) \Rightarrow(2)$, the other being trivial. By the results of Childs and Hurley, if $H$ is commutative, then $H$ satisfies Noether's Theorem.

In this paper we give a general criterion for $H$ to satisfy Noether's Theorem and construct a family of noncommutative Hopf algebras for which this criterion holds. The basic induction techniques used here were first employed by Swan [Swa1], and axiomatized by Lam in the form of Frobenius functors [B, Chapter 9].

This work is adapted from the author's doctoral dissertation at the State University of New York at Albany, completed under the guidance of L. Childs.

\section{Conventions}

All rings will have unit element 1 . Unless explicitly stated otherwise, all module actions are on the left. In any case, we assume 1 acts trivially. All algebras are finitely generated and projective as modules over their base ring and all Hopf algebras are assumed to be cocommutative. Furthermore, unadorned tensoring is over $R$.

\section{HOPF ALGEBRAIC PRELIMINARIES}

Let $R$ be a commutative ring and let $H$ be an $R$-Hopf algebra. Assume that $H$ is finitely generated and projective as an $R$-module. Denote the structure maps of $H$ by $\mu$ (multiplication), $\eta$ (unit), $\Delta$ (comultiplication), $\varepsilon$ (counit), and $\lambda$ (antipode).

We use the Sweedler notation for comultiplication:

$$
\Delta(h)=\sum_{(h)} h_{(1)} \otimes h_{(2)} .
$$

As an example, if $G$ is a finite group, then the group algebra $R G$ is a Hopf algebra with coalgebra structure given by $\Delta(g)=g \otimes g, \varepsilon(g)=1$, and antipode map given by $\lambda(g)=g^{-1}$, for $g \in G$.

If $A$ is an $R$-algebra and an $H$-module, then $A$ is called an $H$-module algebra if the action satisfies the "measuring property" [see Swe], that is, if

$$
h \cdot(a b)=\sum_{(h)}\left(h_{(1)} \cdot a\right)\left(h_{(2)} \cdot b\right) \quad \text { and } \quad h \cdot 1=\varepsilon(h) 1
$$

for all $h \in H$ and $a, b \in A$. For example, if $L / K$ is a Galois extension of fields with group $G$, then $L$ is a $K G$-module algebra.

If $A$ is an $H$-module algebra, then we may form the smash product $A \# H$. As an $R$-module, $A \# H=A \otimes H$, though we write a generator of $A \# H$ as $a \# h$. The algebra structure of the smash product is given by

$$
(a \# h)(b \# g)=\sum_{(h)} a\left(h_{(1)} \cdot b\right) \#\left(h_{(2)} g\right) .
$$

It is easily verified that $A \# H$ is an $R$-algebra with unit element $1 \# 1$. 
Assume now that $K$ is a local field with uniformizing parameter $\pi$, ring of integers $R$, and residue field characteristic $p$. Also, suppose that $R$ contains $\zeta$, a primitive $p$ th root of unity and that $H$ is an $R$-Hopf algebra of rank $p$ over $R$. Then by [T/O], $H$ must be one of the Tate-Oort algebras $H=H_{b} \cong$ $R[x] /\left\langle x^{p}-b x\right\rangle$, where $b=u \pi^{-(p-1) k}, u$ is a unit in $R$, and $0 \leq k \leq e=$ ramification index of the extension $K / \mathbb{Q}_{p}(\zeta)$. (In the interests of brevity, we omit the description of the comultiplication on $H_{b}$. For such a description, see [T/O].) Furthermore, $H_{b}$ is an $R$-order in $K C_{p}=$ the group algebra of $C_{p}$ over $K$, where $C_{p}=\langle\sigma\rangle$ is the cyclic group of order $p$. That is, $K \otimes H_{b} \cong K C_{p}$ as $K$-Hopf algebras. In fact, denoting the image of $x$ in $H_{b}$ by $\xi$, we can identify the image of $\xi$ in $K C_{p}$ using the equation

$$
\xi=-\pi^{-k} \sum_{m \in \mathbb{F}_{p}^{*}} \chi(m) \sigma^{m}
$$

where $\chi: \mathbb{F}_{p}^{*} \rightarrow \mathbb{Z} / p \mathbb{Z} \subseteq R$ is the unique multiplicative section of the residue $\operatorname{map} \mathbb{Z}_{p} \rightarrow \mathbb{Z}_{p} / p \mathbb{Z}_{p}=\mathbb{F}_{p}$.

\section{4. $K$-THEORETIC PRELIMINARIES}

In this section, we state some basic results in the $K$-theory of Hopf algebras. Since most of the results here are simply Hopf algebraic generalizations of analogous group algebra statements, we only sketch the proofs, referring the interested reader to [Swa1] for the details.

Let $A$ be a ring, not necessarily commutative. The Grothendieck group $\mathscr{G}(A)$ is an abelian group defined by generators and relations as follows: $\mathscr{G}(A)$ has one generator $[M]$ for each isomorphism class $M$ of finitely generated $A$-modules. $\mathscr{G}(A)$ has one relation of the form $[M]=\left[M^{\prime}\right]+\left[M^{\prime \prime}\right]$ for each exact sequence of finitely generated left $A$-modules

$$
0 \rightarrow M^{\prime} \rightarrow M \rightarrow M^{\prime \prime} \rightarrow 0 .
$$

An easy induction argument shows that if

$$
0=X_{0} \leq X_{1} \leq X_{2} \leq \cdots \leq X_{n}=X
$$

is a composition series for an $A$-module $X$, then

$$
[X]=\sum\left[X_{i}\right] \text { in } \mathscr{G}(A) .
$$

Accordingly, if $A$ is Artinian, then the Jordan-Hölder Theorem shows that $\mathscr{G}(A)$ is freely generated by the isomorphism classes of simple $A$-modules. In particular, if $A$ is semisimple, then the rank of $\mathscr{G}(A)$ is simply the number of minimal idempotents in $A$, by Wedderburn's Theorem. We summarize with

(4.1) Theorem. If $A$ is semisimple, then $\mathscr{G}(A)$ is a free abelian group generated by the isomorphism classes of simple A-modules.

The group $\mathscr{P}(A)$ is defined similarly, except that one uses finitely generated projective left $A$-modules. It is well known [Swa2, Lemma 4.1, for example] that for two projective $A$-modules $P$ and $Q,[P]=[Q]$ in $\mathscr{P}(A)$ if and only if $P$ and $Q$ are stably isomorphic, that is, $P \oplus F \cong Q \oplus F$ for some free module $F$. If $R$ is a complete discrete valuation ring and $A$ is an $R$-algebra, finitely generated as an $R$-module, then the Krull-Schmidt Theorem holds for 
$A$ [R2, Exercise Chapter 6]. This means that every finitely generated $A$-module admits a unique decomposition into indecomposable direct summands. It then follows that $[P]=[Q]$ in $\mathscr{P}(A)$ if and only if $P \cong Q$. In this case, $\mathscr{P}(A)$ is a free abelian group generated by the isomorphism classes of indecomposable projective modules. In summary,

(4.2) Theorem. If $R$ is a complete discrete valuation ring and $A$ is an $R$-algebra that is finitely generated as an $R$-module, then $\mathscr{P}(A)$ is freely generated by the isomorphism classes of indecomposable projective A-modules.

We henceforth assume that $R$ is at least a Dedekind domain and that $H$ is an $R$-Hopf algebra, finitely generated and projective as an $R$-module. Under these hypotheses, we have

(4.3) Proposition. $\mathscr{G}(H)$ is a ring.

The multiplication is induced by the tensor product, $[M] \cdot[N]=[M \otimes N]$, where the $H$-module structure on $M \otimes N$ is induced by pulling back the $(H \otimes H)$-module structure via $\Delta$. That is,

$$
h \cdot(m \otimes n)=\sum_{(h)}\left(h_{(1)} \cdot m\right) \otimes\left(h_{(2)} \cdot n\right),
$$

for $h \in H, m \in M$, and $n \in N$. Of course, the unit element for this multiplication is $[R]$ where $R$ is the trivial $H$-module via the counit $\varepsilon: H \rightarrow R$.

The ring structure of $\mathscr{G}(H)$ is compatible with the additive structure of $\mathscr{P}(H)$ in that we have the following.

(4.4) Proposition. $\mathscr{P}(H)$ is a module over $\mathscr{G}(H)$.

The multiplication is induced by tensor product, as above. The proof basically entails showing that if $M$ is any module and $P$ is projective then $M \otimes P$ is projective. This, in turn, reduces to showing that for a free $H$-module $F$, the module $M \otimes F$ is free. For this, it suffices to show that $M \otimes H$ is free. But this essentially the statement of [Theorem 4.1.1 of Swe], which is stated for Hopf algebras over fields, but also holds over arbitrary commutative rings if the Hopf algebra $H$ is finitely generated and projective.

The next theorem forms the basis for much of the work in the next section.

(4.5) Theorem (Swan's Triangle). For any ideal $I$ of $R$, there is a unique map $\psi_{I}: \mathscr{G}(K \otimes H) \rightarrow \mathscr{G}(H / I H)$ such that the following diagram commutes:

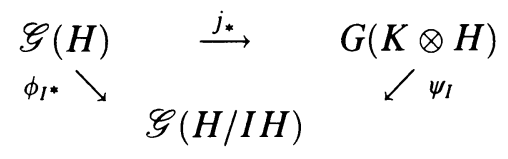

For a proof, see [B, Chapter 10, Proposition 1.1]. Note: the map $j_{*}: \mathscr{G}(H) \rightarrow$ $\mathscr{G}(K \otimes H)$ simply sends a generator $[M]$ to $[K \otimes M]$ and the map $\phi_{I^{*}}: \mathscr{G}(H) \rightarrow$ $\mathscr{G}(H / I H)$ simply send a generator $[M]$ to $[M / I M]$.

By the uniqueness of $\psi_{I}$ and the observation that $\phi_{I^{*}}(1)=1$, we see that $\psi_{I}(1)=1$.

If $J$ is an $R$-Hopf subalgebra of $J$, we say that $J$ is admissible for $H$ if $H$ is finitely generated and projective as both a left and right $J$-module. In this 
case, we obtain maps called, respectively, induction and restriction,

$$
\begin{aligned}
& \operatorname{Ind}_{J}^{H}=\operatorname{Ind}=i_{*}: \mathscr{G}(J) \rightarrow \mathscr{G}(H), \\
& \operatorname{Ind}_{J}^{H}=\operatorname{Ind}=i_{*}: \mathscr{P}(J) \rightarrow \mathscr{P}(H), \\
& \operatorname{Res}_{J}^{H}=\operatorname{Res}=i^{*}: \mathscr{G}(H) \rightarrow \mathscr{G}(J), \\
& \operatorname{Res}_{J}^{H}=\operatorname{Res}=i^{*}: \mathscr{P}(H) \rightarrow \mathscr{P}(J),
\end{aligned}
$$

by defining $\operatorname{Ind}([M])=\left[H \otimes_{J} M\right]$ and $\operatorname{Res}([M])=[M]$.

A well-known induction result in group representation theory is Frobenius Reciprocity (sometimes called the tensor identity). In fact, it holds for Hopf algebra representations, in general.

(4.6) Theorem. If $J$ is an admissible sub-Hopf algebra of $H, M$ is a $J$-module, and $N$ is an $H$-module, then

$$
\left(H \otimes_{J} M\right) \otimes N \cong H \otimes_{J}(M \otimes N),
$$

as $H$-modules where

$\left(H \otimes_{J} M\right) \otimes N$ is an $H$-module via

$$
h \cdot\left(\left(h^{\prime} \otimes m\right) \otimes n\right)=\sum_{(h)}\left(\left(h_{(1)} h^{\prime} \otimes m\right) \otimes\left(h_{(2)} \cdot n\right)\right),
$$

$H \otimes_{J}(M \otimes N)$ is an $H$-module via

$$
h \cdot\left(h^{\prime} \otimes(m \otimes n)\right)=h h^{\prime} \otimes(m \otimes n), \quad \text { and }
$$

$M \otimes N$ is a $J$-module via

$$
j \cdot(m \otimes n)=\sum_{(j)}\left(j_{(1)} \cdot m\right) \otimes\left(j_{(2)} \cdot n\right) .
$$

In the notation introduced above, we have

$$
i_{*}(x) y=i_{*}\left(x i^{*}(y)\right) \quad \text { for all } x \in \mathscr{G}(J), y \in \mathscr{G}(H) .
$$

Proof. The following maps are verified to be inverse $H$-module isomorphisms:

$$
\begin{gathered}
\Phi:\left(H \otimes_{J} M\right) \otimes N \rightarrow H \otimes_{J}(M \otimes N), \\
\Phi((h \otimes m) \otimes n)=\sum_{(h)} h_{(1)} \otimes\left(m \otimes h_{(2)}^{\lambda} \cdot n\right)
\end{gathered}
$$

and

$$
\begin{array}{r}
\Psi: H \otimes_{J}(M \otimes N) \rightarrow\left(H \otimes_{J} M\right) \otimes N, \\
\Psi(h \otimes(m \otimes n))=\sum_{(h)}\left(h_{(1)} \otimes m\right) \otimes h_{(2)} \cdot n .
\end{array}
$$

If $\mathscr{C}$ is a collection of admissible sub-Hopf algebras of $H$, then we obtain an induced map Ind: $\bigoplus \mathscr{G}(J) \rightarrow \mathscr{G}(H)$ where the summation runs over all the sub-Hopf algebras $J$ of $H$ in the collection $\mathscr{C}$. Let $\mathscr{G}_{\mathscr{C}}(H)$ denote the image of this map. We say that $\mathscr{C}$ is cofinite for $H$ if the quotient group $\mathscr{G}(H) / \mathscr{G}_{\mathscr{E}}(H)$ is finite. In this case, there exists an integer $e$ such that $e \mathscr{G}(H) \subseteq \mathscr{G}_{\mathscr{C}}(H)$. We call such an $e$ an (induction) exponent for the collection $\mathscr{C}$. We do not require that $e$ be minimal. With this notation, Frobenius Reciprocity immediately yields an important corollary. 
(4.7) Corollary. $\mathscr{G}_{\mathscr{C}}(H)$ is an ideal in $\mathscr{G}(H)$.

One consequence of Swan's Triangle and Frobenius Reciprocity is that in searching for cofinite collections for $H$ over $R$, it suffices to find collections whose base extensions to $K$ are cofinite for $K \otimes H$. Precisely, we have

(4.8) Theorem. Let $\mathscr{C}$ be an admissible collection of sub-Hopf algebras for $H$ and suppose that $K \otimes \mathscr{C}=\{K \otimes J: J \in \mathscr{C}\}$ is cofinite for $K \otimes \mathscr{C}$ with exponent $e$. Then

(a) For any ideal $I$ of $R, \mathscr{C} / I=\{J / I J: J \in \mathscr{C}\}$ is cofinite for $H / I H$ with exponent $e$.

(b) $\mathscr{C}$ is cofinite for $H$ with exponent $e^{2}$.

Proof. The key observation is that $\mathscr{G}_{\mathscr{C}}$ is an ideal in $\mathscr{G}$. Hence it suffices to show that $e \in \mathscr{G}_{\mathscr{C} / I \mathscr{C}}(H / I H)$ and $e^{2} \in \mathscr{G}_{K \otimes \mathscr{C}}(K \otimes H)$.

(a) Let $\phi_{I}: H \rightarrow H / I H$ be the canonical map and $\phi_{I^{*}}: \mathscr{G}(H) \rightarrow \mathscr{G}(H / I H)$ the induced map. The map $\psi_{I}$ of Swan's Triangle (Theorem 4.5) commutes with induction, hence $\psi_{I}$ maps $\mathscr{G}_{K \otimes \mathscr{C}}(K \otimes H)$ into $\mathscr{G}_{\mathscr{C} / I \mathscr{C}}(H / I H)$. Clearly, $j_{*}(1)=1$. Therefore, $\psi_{I}(1)=\psi_{I}\left(j_{*}(1)\right)=\phi_{I^{*}}(1)=1$. Hence, $e=\psi_{I}(e) \in$ $\psi_{I}\left(\mathscr{G}_{K \otimes \mathscr{C}}(K \otimes H)\right) \subseteq \mathscr{G}_{\mathscr{C} / I \mathscr{C}}(H / I H)$. This proves (a).

(b) First note that extension $j_{*}$ commutes with induction $i_{*}$ from sub-Hopf algebras, hence $j_{*}$ maps $\mathscr{G}_{\mathscr{C}}(H)$ into $\mathscr{G}_{K \otimes \mathscr{C}}(K \otimes H)$. Furthermore, this is surjective by the proof of the exactness of the sequence in [Swa2, Lemma 1.1]. Since $e \in \mathscr{G}_{K \otimes \mathscr{C}}(K \otimes H)$, there is an $x \in \mathscr{G}_{\mathscr{C}}(H)$ such that $j_{*}(x)=e=$ $j_{*}(e)$. By the exact sequence mentioned above, $x-e=\sum \phi_{p}^{*}\left(x_{p}\right)$, where the sum extends over all prime ideals $p$ of $R$ and each $x_{p} \in \mathscr{G}(H / p H)$. If we let $\phi_{p}: H \rightarrow H / p H$ be the canonical map and $\phi_{p}^{*}: \mathscr{G}(H / p H) \rightarrow \mathscr{G}(H)$ the restriction map, we have

$$
e x-e^{2}=\sum e \phi_{p}^{*}\left(x_{p}\right)=\sum \phi_{p}^{*}\left(e x_{p}\right) .
$$

Now exp $\in \mathscr{G}_{\mathscr{C} / p}(H / p H)$ by part (a), so it follows that $\phi_{p}^{*}\left(e x_{p}\right) \in \mathscr{G}_{\mathscr{C}}(H)$ for each $p$. Thus, ex $-e^{2} \in \mathscr{G}_{\mathscr{C}}(H)$. Since $\mathscr{G}_{\mathscr{C}}(H)$ is an ideal in $\mathscr{G}(H)$, $x \in \mathscr{G}_{\mathscr{C}}(H)$ implies that $e x \in \mathscr{G}_{\mathscr{C}}(H)$. Hence, $e^{2} \in \mathscr{G}_{\mathscr{C}}(H)$. This proves (b).

In order to exploit this result, we need some cofinite collections for $K \otimes H$. When $K \otimes H$ is a group algebra $K G$, this is realized with the following wellknown theorem [S, Section 9.2, for example].

(4.9) Theorem (Artin induction). Suppose $G$ is a finite group and $K$ is a field. Let $\mathscr{C}$ be the collection of all subgroup algebras $K C$, where $C$ is a cyclic subgroup of $G$. Then $\mathscr{C}$ is cofinite for $K G$ and the order of $G$ serves as an induction exponent for $\mathscr{C}$.

We finish this section with some general observations about induction in group algebras. Let $K G$ be a group algebra and suppose that $\mathscr{C}$ and $\mathscr{D}$ are collections of subgroup algebras of $K G$. If every $C \in \mathscr{C}$ is contained in some $D \in \mathscr{D}$, we say that the $\mathscr{C}$ is a refinement of $\mathscr{D}$. If every $C \in \mathscr{C}$ has a conjugate contained in some $D \in \mathscr{D}$, we say that $\mathscr{C}$ is a conjugate refinement of $\mathscr{D}$. We then have two observations.

(4.10) Proposition. If $\mathscr{C}$ is cofinite and a refinement of $\mathscr{D}$, then $\mathscr{D}$ is cofinite. 
Proof. This is simply transitivity of induction. Let $e$ be an induction exponent for $\mathscr{C}$ and let $C \subseteq D$ with $C \in \mathscr{C}$ and $D \in \mathscr{D}$. Then for any $K C$-module $M$, we have

$$
\operatorname{Ind}_{K C}^{K G}(M) \cong \operatorname{Ind}_{K D}^{K G}\left(\operatorname{Ind}_{K C}^{K D}(M)\right) .
$$

It follows that $\mathscr{G}_{\mathscr{C}}(K G) \subseteq \mathscr{G}_{\mathscr{D}}(K G)$. Therefore, $e \mathscr{G}(K G) \subseteq \mathscr{G}_{\mathscr{C}}(K G) \subseteq \mathscr{G}_{\mathscr{D}}(K G)$. Hence, $\mathscr{D}$ is cofinite.

(4.11) Lemma. If $C$ and $D$ are conjugate subgroups of $G$, then

$$
\operatorname{Ind}_{K C}^{K G}: \mathscr{G}(K C) \rightarrow \mathscr{G}(K G) \text { and } \operatorname{Ind}_{K D}^{K G}: \mathscr{G}(K D) \rightarrow \mathscr{G}(K G)
$$

have the same image.

Proof. In fact, we show that every $K G$-module induced from a $K C$ is isomorphic to a module induced from $K D$. Let $C=\tau D \tau^{-1}, \tau \in G$, and suppose that $M$ is a $K C$-module. Define a $K D$-module structure on $M$ by letting $\delta: m=\left(\tau \delta \tau^{-1}\right) \cdot m$ for $\delta \in D$ and $m \in M$. We define a map

$$
\begin{gathered}
\psi: K G \otimes_{K C} M \rightarrow K G \otimes_{K D} M, \\
\sigma \otimes m \mapsto \sigma \tau \otimes m .
\end{gathered}
$$

This is clearly a $K G$-linear isomorphism, provided that it is well defined. We need to show that $\psi(\sigma \gamma \otimes m)=\psi(\sigma \otimes \gamma m)$ for all $\sigma \in G, \gamma \in C$, and $m \in M$. Computing,

$$
\begin{aligned}
\psi(\sigma \gamma \otimes m) & =\sigma \gamma \tau \otimes m=\sigma \tau \delta \otimes m, \text { for some } \delta \in D \\
& =\sigma \tau \otimes \delta: m=\sigma \tau \otimes\left(\tau \delta \tau^{-1}\right) \cdot m \\
& =\sigma \tau \otimes \gamma m=\psi(\sigma \otimes \gamma m), \quad \text { as required. }
\end{aligned}
$$

(4.12) Corollary. If $\mathscr{C}$ is cofinite and a conjugate refinement of $\mathscr{D}$, then $\mathscr{D}$ is cofinite.

\section{NOETHER'S THEOREM}

In this section we determine a general criterion for Noether's Theorem to hold for a Hopf algebra. We then construct a family of nontrivial noncommutative Hopf algebras and use the criterion to show that Noether's Theorem holds for this family. In this context, nontrivial means not a group algebra.

Let $R$ be a discrete valuation ring with quotient field $K$, and let $H$ be an $R$-Hopf algebra, finitely generated and projective as an $R$-module. We say that Noether's Theorem holds for $H$ if the following are equivalent:

(1) $S$ is a projective $H$-module and $K \otimes S \cong K \otimes H$ as $K \otimes H$-modules.

(2) $S \cong H$ as $H$-modules.

The only implication of interest here is $(1) \Rightarrow(2)$, the other being trivial. By the results of Childs and Hurley [C/H, Theorem 5.2], if $H$ is commutative, then $H$ satisfies Noether's Theorem.

It is a technical simplification to assume that $R$ is complete. This is no loss of generality because two $H$-modules are isomorphic if and only if their completions are isomorphic $[\mathrm{R} 1, \S 1,(19)]$. Thus, we may assume $R$ complete and then the Krull-Schmidt Theorem holds for $H$-modules (cf. comment preceding Theorem 4.2). 
(5.1) Proposition. The following are equivalent (recall that $j: R \rightarrow K$ is the inclusion):

(a) $j_{*}: \mathscr{P}(H) \rightarrow \mathscr{G}(K \otimes H)$ is injective.

(b) If $M, N$ are projective $H$-modules and $K \otimes M \cong K \otimes N$ as $K \otimes H$ modules, then $M \cong N$ as $H$-modules.

Proof. (a) $\Rightarrow$ (b): Suppose that $j_{*}$ is injective and $M, N$ are projective $H$ modules with $K \otimes M \cong K \otimes N$ as $K \otimes H$-modules. Then $j_{*}([M])=j_{*}([N])$. By injectivity, we have $[M]=[N]$. But now Theorem 4.2 implies $M \cong N$.

(b) $\Rightarrow$ (a): If $x=[M]-[N] \in \operatorname{ker} j_{*}$, then $[K \otimes M]=[K \otimes N]$. Since $K \otimes H$ is semisimple, we have $K \otimes M \cong K \otimes N$. By the assumption (b), we have that $M \cong N$ and therefore $x=[M]-[N]=0$. Hence, $j_{*}$ is injective.

(5.2) Corollary. If $j_{*}: \mathscr{P}(H) \rightarrow \mathscr{G}(K \otimes H)$ is injective, then Noether's Theorem holds for $H$.

The map $j_{*}: \mathscr{P}(H) \rightarrow \mathscr{G}(K \otimes H)$ is injective, hence Noether's Theorem holds for $H$, if $H$ is commutative. For a proof, see [Swa1, Lemma 6.2] or $[\mathrm{C} / \mathrm{H}$, Theorem 5.2]. We now show that if $H$ is rich enough in the right kind of admissible sub-Hopf algebras, then Noether's Theorem holds for $H$.

(5.3) Theorem. Noether's Theorem holds for $H$ if and only if $H$ has a cofinite collection $\mathscr{C}$ of admissible sub-Hopf algebras such that $j_{*}: \mathscr{P}(J) \rightarrow \mathscr{G}(K \otimes J)$ is injective for all $J \in \mathscr{C}$.

Proof. The necessity is obvious for if Noether's Theorem holds for $H$ then the collection $\mathscr{C}=\{H\}$ does the job. For the sufficiency, it suffices to show that $j_{*}: \mathscr{P}(H) \rightarrow \mathscr{G}(K \otimes H)$ is injective.

Let $x \in \mathscr{P}(H)$ and suppose that $j_{*}(x)=0$. Let $N$ be an exponent for the collection $\mathscr{C}$. Then we have

$$
N=\sum i_{\nu^{*}}\left(x_{\nu}\right) \text { in } \mathscr{G}(H),
$$

where $x_{\nu} \in \mathscr{G}\left(J_{\nu}\right), J_{\nu} \in \mathscr{C}$, and $i_{\nu}: J_{\nu} \rightarrow H$ is the inclusion map and $i_{\nu^{*}}: \mathscr{G}\left(J_{\nu}\right) \rightarrow \mathscr{G}(H)$ is the induction map. By admissibility of $J_{\nu}$, we have $i_{\nu}^{*}(x) \in \mathscr{P}\left(J_{\nu}\right)$. (Recall that $i_{\nu}^{*}: \mathscr{G}(H) \rightarrow \mathscr{G}\left(J_{\nu}\right)$ is the homomorphism induced by restriction.) Since $\mathscr{P}\left(J_{\nu}\right)$ is a module over $\mathscr{G}\left(J_{\nu}\right)$, we have $x_{\nu} i_{\nu}^{*}(x) \in \mathscr{P}\left(J_{\nu}\right)$ and the following equalities in $\mathscr{G}\left(K \otimes J_{\nu}\right)$ for each $\nu$ :

$$
\begin{aligned}
j_{*}\left(x_{\nu} i_{\nu}^{*}(x)\right) & =j_{*}\left(x_{\nu}\right) j_{*}\left(i_{\nu}^{*}(x)\right)=j_{*}\left(x_{\nu}\right) i_{\nu}^{*}\left(j_{*}(x)\right) \\
& =j_{*}\left(x_{\nu}\right) i_{\nu}^{*}(0)=j_{*}\left(x_{\nu}\right) 0=0 .
\end{aligned}
$$

By hypotheses $j_{*}: \mathscr{P}\left(J_{\nu}\right) \rightarrow \mathscr{G}\left(K \otimes J_{\nu}\right)$ is injective. Therefore, $x_{\nu} i_{\nu}^{*}(x)=0$. Now we have the following in $\mathscr{P}(H)$ :

$$
\begin{aligned}
N x & =\sum i_{\nu^{*}}\left(x_{\nu}\right) x \\
& =\sum i_{\nu^{*}}\left(x_{\nu} i_{\nu}^{*}(x)\right), \quad \text { by Frobenius Reciprocity }(4.6) \\
& =\sum i_{\nu^{*}}(0)=0 .
\end{aligned}
$$

So $N x=0$ in $\mathscr{P}(H)$. But $\mathscr{P}(H)$ is a free abelian group, by Theorem 4.2. Therefore, $x=0$. This proves that $j_{*}: \mathscr{P}(H) \rightarrow \mathscr{G}(K \otimes H)$ is injective.

We now construct our family of Hopf algebras for which Noether's Theorem holds. Let $C_{p}=\langle\sigma\rangle$ be a cyclic group of prime order $p$, let $C_{n}=\langle\tau\rangle$ be a cyclic 
group of order $n$ and suppose that $C_{n}$ acts faithfully on $C_{p}$ via $=\tau \cdot \sigma=\sigma^{\alpha}$, where $\alpha \in \mathbb{F}_{p}^{*}=(\mathbb{Z} / p \mathbb{Z})^{*}$. Let $G=C_{p} C_{n}$ denote the semidirect product of $C_{p}$ and $C_{n}$ via this action. Let $K$ be a local field with valuation ring $R$ and maximal ideal $\pi R$ containing $p$. Also assume that $\zeta=\zeta_{p}$ is a primitive $p$ th root of unity in $R$.

Let $\chi: \mathbb{F}_{p}^{*} \rightarrow \mathbb{Z} / p \mathbb{Z} \subseteq R$ be the unique multiplicative section of the residue $\operatorname{map} \mathbb{Z}_{p} \rightarrow \mathbb{Z}_{p} / p \mathbb{Z}_{p}=\mathbb{F}_{p}$

Let $H_{b}, b=u \pi^{k(p-1)}$, be a Tate-Oort Hopf algebra order in $K C_{p}$ with algebra generator

$$
\xi=-\pi^{-k} \sum_{m \in \mathbb{F}_{p}^{*}} \chi(m) \sigma^{m}
$$

The action of $C_{n}$ on $C_{p}$ makes $K C_{p}$ into a $K C_{n}$-module algebra and we may form the smash product

$$
K C_{p} \# K C_{n} \cong K\left[C_{p} C_{n}\right] \cong K[G]
$$

(5.4) Proposition. Under the restricted action, $H_{b}$ is an $R C_{n}$-module algebra.

Proof. The measuring property follows from the measuring property over $K$. We merely need to show that $H_{b}$ is invariant under the action of $R C_{n}$. By the measuring property, it suffices to check that the algebra generator $\xi$ of $H_{b}$ is mapped back into $H_{b}$ by $\tau$.

$$
\begin{aligned}
\tau \cdot \xi & =\tau \cdot\left(-\pi^{-k} \sum_{x \in \mathbb{F}_{p}^{*}} \chi(x) \sigma^{x}\right)=-\pi^{-k} \sum_{x \in \mathbb{F}_{p}^{*}} \chi(x) \tau \cdot\left(\sigma^{x}\right) \\
& =-\pi^{-k} \sum_{x \in \mathbb{F}_{p}^{*}} \chi(x) \sigma^{\alpha x}=-\pi^{-k} \sum_{y \in \mathbb{F}_{p}^{*}} \chi\left(\alpha^{-1} y\right) \sigma^{y} \\
& =\chi\left(\alpha^{-1}\right)\left(-\pi^{-k} \sum_{y \in \mathbb{F}_{p}^{*}} \chi(y) \sigma^{y}\right)=\chi\left(\alpha^{-1}\right) \xi
\end{aligned}
$$

That is, $\tau \cdot \xi=\chi\left(\alpha^{-1}\right) \xi \in H_{b}$, as required.

Since $H_{b}$ is $R C_{n}$-invariant, we may form the smash product $H=H_{b} \# R C_{n}$. Observe that extending the base to $K$ yields

$$
\begin{aligned}
K \otimes H & =K \otimes\left(H_{b} \# R C_{n}\right) \cong\left(K \otimes H_{b}\right) \otimes_{K}\left(K \otimes R C_{n}\right) \\
& \cong K C_{p} \otimes_{K} K C_{n} \cong K\left[C_{p} C_{n}\right] \cong K[G] .
\end{aligned}
$$

So $H$ is a nontrivial noncommutative Hopf algebra order in the group algebra $K G$. Furthermore, $H$ contains the two commutative admissible sub-Hopf algebras $H_{b}$ and $R C_{n}$.

To show that $\left\{H_{b}, R C_{n}\right\}$ is cofinite for $H$, it suffices to show that $\left\{K C_{p}\right.$, $\left.K C_{n}\right\}$ is cofinite for $K G$, by Theorem 4.8. That is, we may show that the collection $\mathscr{C}=\left\{C_{p}, C_{n}\right\}$ is cofinite for the group $G=C_{p} C_{n}$ over the field $K$.

We record the following properties of $G$ as a lemma, whose proof is straightforward.

(5.5) Lemma. Let $G=C_{p} C_{n}$, with $\tau \sigma \tau^{-1}=\sigma^{\alpha}, \alpha \in \mathbb{F}_{p}^{*}$.

(a) Every element $x \in G$ may be expressed in the form $x=\sigma^{s} \tau^{t}$, with $s \in \mathbb{Z} / p \mathbb{Z}, t \in \mathbb{Z} / n \mathbb{Z}$. 
(b) $\tau^{i} \sigma^{j}=\sigma^{\alpha^{i}} \tau^{j}$

(c) $\left(\sigma^{u} \tau^{v}\right)^{-1}=\sigma^{-u \alpha^{-1}} \tau^{-v}$.

(d) $\left(\sigma^{u} \tau^{v}\right)\left(\sigma^{s} \tau^{t}\right)\left(\sigma^{u} \tau^{v}\right)^{-1}=\sigma^{u+\alpha^{v} s-\alpha^{v} u} \tau^{v}$.

(5.6) Proposition. Every cyclic subgroup $C$ of $G$ (except the normal subgroup $C_{p}$ itself) is conjugate to a subgroup of $C_{n}=\langle\tau\rangle$.

Proof. This is just a computation using the lemma. If $\sigma^{s} \tau^{t}$ is a generator for $C$, we seek $u$ and $v$ such that

$$
\left(\sigma^{u} \tau^{v}\right)\left(\sigma^{s} \tau^{t}\right)\left(\sigma^{u} \tau^{v}\right)^{-1}=\tau^{t} \in\langle\tau\rangle=C_{n}
$$

But the left side reduces to $\sigma^{u+\alpha^{v} s-\alpha^{t} u} \tau^{v}=\tau^{t}$. It suffices to take $v=0$. This leaves us with the task of solving the congruence

$$
u+s-\alpha^{t} u \equiv 0 \quad(\bmod p)
$$

or

$$
u\left(\alpha^{t}-1\right) \equiv s \quad(\bmod p) .
$$

But since $\sigma^{s} \tau^{t} \notin C_{p}$, we have $\tau^{t} \neq 1$. This implies that $\tau^{t}$ acts nontrivially on $\sigma$, by the faithfulness of the action of $C_{n}$ on $C_{p}$. But $\tau^{t} \cdot \sigma=\sigma^{\alpha^{t}}$ so $\alpha^{t} \not \equiv 1(\bmod p)$. Therefore, $\alpha^{t}-1$ is a unit $\bmod p$ and we may solve for $u=s /\left(\alpha^{t}-1\right) \in \mathbb{Z} / p \mathbb{Z}$. This completes the proof.

(5.7) Proposition. $C_{p}=\langle\sigma\rangle$ is the only abelian subgroup of $G$ whose order is divisible by $p$.

Proof. Let $A$ be any such subgroup. Since $p$ divides the order of $A$, it must have an element of order $p$, by Sylow's Theorem. But the only elements of order $p$ are in $C_{p}$ and any one of these generates $C_{p}$ so $C_{p} \leq A$. If $\sigma^{s} \tau^{t} \in A$, then $\tau^{t} \in A$. It follows $\tau^{t}$ commutes with every element of $C_{p}$. By the faithfulness of the action, we must have $\tau^{t}=1$. Therefore, $A \leq C_{p}$. Hence, $A=C_{p}$.

We now state the main theorem of this section.

(5.8) Theorem. Noether's Theorem holds for $H=H_{b} \# R C_{n}$.

Proof. By Artin's Theorem, the collection of cyclic subgroups of $G$ is cofinite for $G$. By Propositions 5.6 and 5.7, we see that the collection $\left\{C_{p}, C_{n}\right\}$ is a conjugate refinement for the collection of cyclic subgroups of $G$, hence is also cofinite for $G$, by Proposition 4.12. By Theorem 4.8, this implies that $\left\{H_{b}, R C_{n}\right\}$ is cofinite for $H$. Hence, Noether's Theorem holds for $H$, by Theorem 5.3.

Application. If $f(x) \in K[x]$ is a solvable polynomial of prime degree $p$, then the Galois group of $f$ (that is, the Galois group $G$ of the extension $L / K$, where $L$ is a splitting field for $f$ ) is a subgroup of the holomorph of $C_{p}$, $\operatorname{Hol}\left(C_{p}\right)=C_{p} \operatorname{Aut}\left(C_{P}\right) \cong C_{p} C_{n}, n=p-1$. For simplicity, assume that $G=$ $C_{p} C_{n}$ and let $H=H_{b} \# R C_{n}$ be a Hopf order in $K G$ as before. Let $S$ be the valuation ring of $L$ and following [Ta], set $T=\{t \in S: H t \subseteq S\}$. Then $T$ is clearly an $H$-module and by the measuring property of the $H$-action, $T$ is a subring of $S$. In fact, $T$ is an $H$-module algebra order in $L$ so we have 
as $H$-modules. the isomorphism $L \cong K G$ deriving from the classical normal basis theorem.

To apply Noether's Theorem to the extension $T / R$, it remains to show that $T$ is $H$-projective. Let

$$
U=T^{H_{b}}=\left\{t \in T: h t=\varepsilon(h) t \text { for all } h \in H_{b}\right\},
$$

the fixed ring of $T$ under $H_{b}$. Since $H_{b}$ is generated as an algebra by $\xi$ and $\varepsilon(\xi)=0$, it follows that $U=\{t \in T: \xi t=0\}$.

(5.9) Proposition. $U$ is invariant under the action of $R C_{n}$ on $L$.

Proof. Let $u \in U$. We need to show that $\tau u \in U$, that is, we must show that $\xi(\tau u)=0$. But

$$
\begin{aligned}
\xi(\tau u) & =\left(-\pi^{-k} \sum_{x \in \mathbb{F}_{p}^{*}} \chi(x) \sigma^{x}\right) \cdot(\tau u) \\
& =\left(-\pi^{-k} \sum \chi(x) \sigma^{x} \tau\right) u \\
& =\left(-\pi^{-k} \sum \chi(\alpha y) \sigma^{\alpha y} \tau\right) u \\
& =\chi(\alpha)\left(-\pi^{-k} \sum \chi(y) \tau \sigma^{y}\right) u \\
& =\chi(\alpha) \tau \xi(u)=0
\end{aligned}
$$

since $u \in U$. That is, $\xi(\tau u)=0$ so $\tau u \in U$, proving that $U$ is $R C_{n}$-invariant.

Now we have an exact sequence of Hopf algebras

$$
0 \rightarrow H_{b} \rightarrow H \rightarrow R C_{n} \rightarrow 0 \text {. }
$$

By [G], the extension $T / R$ is $H$-tame (i.e., $I_{H} T=R$, where $I_{H}$ is the set of integrals in $H)$ if and only if $T / U$ is $\left(U \otimes H_{b}\right)$-tame and $U / R$ is $R C_{n^{-}}$tame. The integrals of $R C_{n}$ are generated by $\sum \tau^{i}$. Since $n=p-1$ is a unit in $R$, the integral $\frac{1}{n} \sum \tau^{i}$ of $R C_{n}$ maps $1 \in U$ to $1 \in R$. This shows that $I_{R C} U=R$, so $U / R$ is $R C_{n}$-tame. Thus, $T / R$ is $H$-tame if and only if $T / U$ is $\left(U \otimes H_{b}\right)$-tame.

Let $E=K \otimes U=K \otimes\left(T^{H_{b}}\right)=(K \otimes T)^{K \otimes H_{b}}=L^{K C_{p}}=L^{C_{p}}$. Then $L / E$ is a cyclic extension of degree $p$ and since $\zeta_{p} \in E, L / E$ is a Kummer extension.

Accordingly, let $L=E[z], z^{p}=w \in U$, where the action of $C_{p}$ on $L$ is given by $\sigma(z)=\zeta z$. We also assume that $w=1+r \pi_{1}^{k p+1}$ is a unit in $R$, where $\pi_{1}$ is a uniformizer for $U, r$ is a unit in $U$ and our $H_{b}$ is the Tate-Oort algebra with $b=u \pi^{-k(p-1)}$. With this assumption, $T / U$ is $\left(U \otimes H_{b}\right)$-Galois [C, Theorem 14.3], hence $\left(U \otimes H_{b}\right)$-tame [C/H, Proposition 2.3]. It follows that $T / R$ is $H$-tame and hence $T$ is projective as an $H$-module $[\mathrm{C} / \mathrm{H}$, Theorem 5.1].

We may now apply Noether's Theorem to conclude that $T \cong H$ as $H$ modules.

\section{REFERENCES}

[B] H. Bass, Algebraic $K$-theory, Benjamin, 1968.

[C1] L. N. Childs, Taming wild extensions with Hopf algebras, Trans. Amer. Math. Soc. 304 (1987), 111-140. 
[C2] L. N. Childs and S. Hurley, Tameness and local normal basis for objects of finite Hopf algebras, Trans. Amer. Math. Soc. 298 (1986), 763-778.

[G] C. Greither, Hopf Galois structure on extensions of local number rings, (preprint).

[K/C] H. F. Kreimer and P. M. Cook, Galois theories and normal basis, J. Algebra 43 (1976), 115-121.

[N] E. Noether, Normalbasis bei Korpern ohne hohere Verzweigung, Crelle, 1931.

[P] B. Pareigis, When Hopf algebras are Frobenius algebras, J. Algebra 18 (1971), 588-596.

[R1] I. Reiner, Integral representations, Lecture Notes in Math., vol. 744, Springer-Verlag, 1979.

[R2] - Maximal orders, Academic Press, 1975.

[S] J.-P. Serre, Integral representations of finite groups, Graduate Texts in Math., no. 42, Springer, 1977.

[Swa1] R. Swan, Induced representations and projective modules, Ann. of Math. (2) 71 (1960), 552-578.

[Swa2] __, The Grothendieck ring of a finite group, Topology 2 (1963), 85-110.

[Swe] M. E. Sweedler, Hopf algebras, Benjamin, 1972.

[T/O] J. Tate and F. Ort, Group schemes of prime order, Ann. Sci. Ecole Norm. Sup. (4) 3 (1970).

[Ta] M. Taylor, Hopf structure and the Kummer theory of formal groups, J. Reine Angew. Math. 375/376 (1987), 1-11.

[W] W. Waterhouse, Tame objects for finite commutative Hopf algebras, Proc. Amer. Math. Soc. 103 (1988), 354-356.

Department of Mathematics and Computer Science, Russell Sage College, Troy, New YORK 12180 